\title{
Effect of organic-phase solvents on physicochemical properties and cellular uptake of astaxanthin nanodispersions.
}

\begin{abstract}
A simplex centroid mixture design was used to study the interactions between two chosen solvents, dichloromethane (DCM) and acetone (ACT), as organic-phase components in the formation and physicochemical characterization and cellular uptake of astaxanthin nanodispersions produced using precipitation and condensation processes. Full cubic or quadratic regression models with acceptable determination coefficients were obtained for all of the studied responses. Multiple-response optimization predicted that the organic phase with $38 \%(\mathrm{w} / \mathrm{w})$ DCM and $62 \%(\mathrm{w} / \mathrm{w})$ ACT yielded astaxanthin nanodispersions with the minimum particle size $(106 \mathrm{~nm})$, polydispersity index $(0.191)$, and total astaxanthin loss $(12.7 \%, \mathrm{w} / \mathrm{w})$ and the maximum cellular uptake $(2981 \mathrm{fmol} / \mathrm{cell})$. Astaxanthin cellular uptake from the produced nanodispersions also showed a good correlation with their particle size distributions and astaxanthin trans/cis isomerization ratios. The absence of significant ( $\mathrm{p}>$ 0.05) differences between the experimental and predicted values of the response variables confirmed the adequacy of the fitted models.
\end{abstract}

Keyword: Astaxanthin nanodispersions; Cellular uptake; Organic-phase solvents; Physicochemical characteristics. 